\title{
Preservation of Historical Documents using ICM and Filters
}

\author{
Ashika Parvin ${ }^{1}$, Shajun Nisha ${ }^{2}$, Mohamed Sathik ${ }^{3}$ \\ M. Phil (Scholar), Computer Science, Sadakathullah Appa College, Tirunelveli, India ${ }^{1}$ \\ Prof \& Head, PG Department of Computer Science, Sadakathullah Appa College, Tirunelveli, India ${ }^{2}$ \\ Principal, Sadakathullah Appa College, Tirunelveli, India ${ }^{3}$
}

\begin{abstract}
Digital image enhancement technique is used for improving the visual qualities of the images. In this paper, contaminated Brahmi script is taken as an input and it is enhanced. There are numerous problems in preserving historical documents from degradation due to some bad storage conditions and poor contrast due to humidity. In this work, it is proposed to investigate the efficiency of logarithmic technique to enhance along with the performance of utilizing the Intersecting Cortical Model (ICM) network which is a simplified model of Pulse-Coupled Neural Network (PCNN) model and filters.
\end{abstract}

Keywords: Digital Image Enhancement, PCNN, Filters, Brahmi script.

\section{INTRODUCTION}

Image processing is a common term that covers image segmentation, image registration, image fusion, image thinning, image enhancement, edge detection, feature extraction, image recognition, noise removal from image, classification of images, texture and fabric defects identification, and surveillance. Image Enhancement is the improvement of digital image quality, without knowledge about the source of degradation. Enhancement may be used to restore an image that has suffered some kind of deterioration due to the optics, electronics and environment. The manuscripts are the hand written documents and the techniques of writing manuscripts are called as "Paleography". These scripts get damaged or erased due to the calamities of time and nature, the poor quality of paper, dirt, humidity or usage of poor quality inks.To enhance certain features of an image Logarithmic transformation method is used. It is used to brighten the intensities of an image More often, it is used to increase the contrast of lower intensity values. Pulse coupled neural network (PCNN) can remove contamination of scripts when used along with the application of filters. Pulse coupled neural network (PCNN) is a single-layer, two-dimensional algorithm is laterally connected network of integrate-andfire neurons between the image pixels and network neurons. It is a good pre-processor in which neurons produces temporal series of pulse outputs and has the following advantages like global optimal approximation characteristic and favorable classification capability, rapid convergence of learning procedure, an optimal network to accomplish the mapping function in the feed-forward, no need to pre-train. The Intersecting Cortical Model (ICM), or ICM algorithm presented is a reduced set of equations of the Pulse-Coupled Neural Network (PCNN) model. Filtering is a technique for modifying or enhancing an image to emphasize certain features or remove other features which includes smoothing, sharpening, and edge enhancement.

\section{RELATED WORK}

Digital image processing is a broad subject and often involves procedures which can be mathematically complex, but central idea behind digital image processing is quite simple[1]. Generally image enhancement techniques are used to get detail that is obscured, or to highlight certain features of interest in image. In image enhancement process one or more attributes of image are modified. In spatial domain techniques [2], we directly deal with the image pixels. The pixel values are manipulated to achieve desired enhancement. These enhancement operations are performed in order to modify the image brightness, contrast or the distribution of the grey levels.. One such method of transformation used in this paper is log transformation maps [3] a narrow range of low input grey level values into a wider range of output values. Log functions are particularly useful when the input grey level values may have an extremely large range of values. This technique was used by[4]. By using this log transformation darker pixel values of the image are expanded by compressing the values in the higher levels. Compression of dynamic range values in an image by giving large variation in the pixel value is considered as the main characteristics of log transformation. Image enhancement using $\log$ transformation can only improve the quality of an image. But for betterment we combine it with pulse-coupled neural network (PCNN) model is used along with filters. In the late 1980s, Eckhorn et al. discovered that the midbrain in an oscillating way created binary images that could extract different features from the visual impression when they 
had studied the cat visual cortex [6-8]. Based on these binary images the actual image is created in the cat brain. Due to this discovery they developed a neural network, called Eckhorn's model, to simulate this behavior.In the early 1990s, Rybak et al. also found the similar neural behavior based on the study of the visual cortex of the guinea pig and developed a neural network, called Rybak's model $[9,10]$. Because Eckhorn's model and Rybak' model provided a simple, effective way for studying synchronous pulse dynamics in networks, they was recognized as being very potential in image processing [11-13]. The functioning of the visual cortex has to be studied in order to develop algorithms. This is more complicated than programming of computers. Researchers started working in the beginning of 1950s [13]. Pulse coupled neural networks are unsupervised networks, in which the network is provided with inputs but not the desired outputs. The network is self organized.

\section{MOTIVATION AND JUSTIFICATION}

Linear Transformation has an important characteristic that it preserves relative spacings. Values that are evenly spaced before transformation remain evenly spaced after transformation. So better enhancement cannot be achieved using this. To overcome this problem log transformation is used to an image which will expand its low valued pixels to a higher level and has little effect on higher valued pixels so in other words it enhances image in such a way that it highlights minor details of an image. When used log transformation along with pulse-coupled neural network (PCNN) intersecting cortical model (ICM) it works better because ICM algorithm, is a reduced set of equations of the Pulse-Coupled Neural Network (PCNN) model where there are no linking neurons. This is especially designed for enhancing features without sharp edges or straight lines in images. It is faster than the pulse-coupled neural network (PCNN) and hence it may be preferable to be used in many cases. Motivated by all these facts, we are inspired to increase the quality of an image using $\log$ transformation and ICM algorithm. Hence I justify that the association of enhancement techniques with Intersecting Cortical Model (ICM), a self-organized network and filtering technique is suitable for this application.

\section{ORGANIZATION OF THE PROPOSED WORK}

The remaining paper is organized as follows: Section V includes Methodology which includes the outline of the proposed work, Section VI includes the Experimental results, Section VII includes Performance Evaluation, Section VIII includes conclusion of the paper.

\section{METHODOLOGY}

In this paper, a contaminated brahmi script is taken as an input for which enhancement technique is used for getting rid of some deteriorations that are present in the image. Logarithmic transformation is one of the enhancement technique that is used to brighten the image. It is a pre-processing step in this work. The process then continues with applying PCNN algorithm to overcome contamination in an image. Once they have been removed they can be enhanced more using filters. The main advantage of filters is re-evaluating the value of every pixel in an image to reduce noise by smoothing, and/or enhance edges. Filters provide an aid to visual interpretation of images. The image that is reconstructed is free from noise.

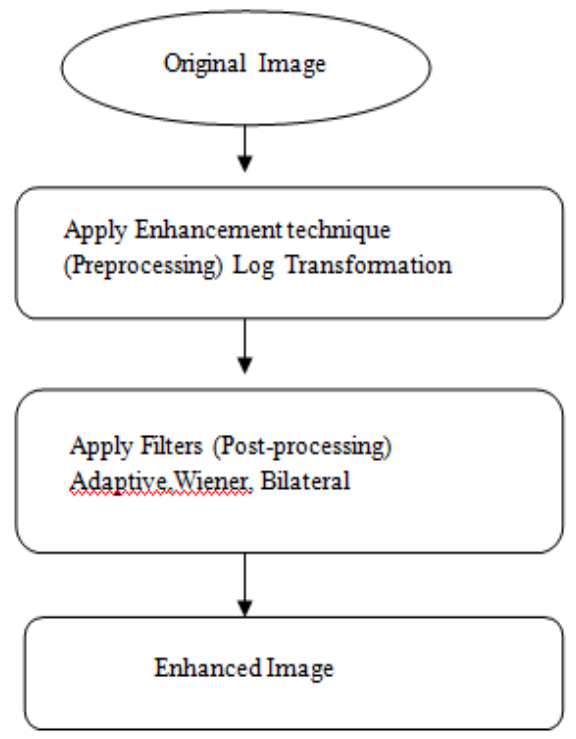

Fig 1.Outline of proposed work 


\section{a) Logarithmic Transformation}

Image transformation can be done by logarithmic transformation to make highly skewed distributions less skewed. This can be valuable both for making patterns in the data more interpretable and for helping to meet the assumptions of inferential statistics. Log transformation in general in shown by this equation:

$$
\mathrm{s}=\mathrm{c} \log (1+\mathrm{r})
$$

where $\mathrm{s}$ and $\mathrm{r}$ are the pixel values of the output and the input image and $\mathrm{c}$ is a constant. It can have intensity range from 0 to $10^{\wedge} 6$ or higher.

\section{b) Pulse-Coupled Neural Networks (PCNN)}

Over the past decade, PCNNs have been used in a variety of image processing applications, including image segmentation feature generation, face extraction, motion detection, region growing, and noise reduction. A PCNN is a two-dimensional neural network. Each neuron in the network corresponds to one pixel in an input image, receiving its corresponding pixel's color information (e.g. intensity) as an external stimulus. Each neuron also connects with its neighboring neurons, receiving local stimuli from them. Through iterative computation, PCNN neurons produce temporal series of pulse outputs. The standard PCNN model is described as iteration by the following equations :

$$
\begin{aligned}
& F_{i, j}[n]=e^{-\alpha_{F}} F_{i, j}[n-1]+V_{F} \sum_{k, l} w_{i, j, k, 1} Y_{i, j}[n-1]+S_{i, j} \\
& L_{i, j}[n]=e^{-\alpha_{1}} L_{i, j}[n-1]+V_{L} \sum_{k, l} m_{i, j, k, 1} Y_{i, j}[n-1] \\
& U_{i, j}[n]=F_{i, j}[n]\left(1+\beta L_{i, j}[n]\right) \\
& Y_{i, j}[n]= \begin{cases}1 & U_{i, j}[n]>T_{i, j}[n] \\
0 & \text { otherwise }\end{cases} \\
& T_{i, j}[n]=e^{-\alpha_{T}} T_{i, j}[n-1]+V_{T} Y_{i, j}[n]
\end{aligned}
$$

In these equations, $\mathrm{S}[\mathrm{i}, \mathrm{j}]$ is the input stimulus such as the normalized gray level of image pixels in $[\mathrm{i}, \mathrm{j}]$ position, $\mathrm{F}_{\mathrm{ij}}[\mathrm{n}]$ is the feedback input of the neuron in $(\mathrm{i}, \mathrm{j})$ and $\mathrm{L}_{\mathrm{ij}}[\mathrm{n}]$ is the linking item. $\mathrm{U}_{\mathrm{ij}}[\mathrm{n}]$ is the internal activity of neuron, and, $\mathrm{T}_{\mathrm{ij}}[\mathrm{n}]$ is the dynamic threshold. $\mathrm{Y}_{\mathrm{ij}}[\mathrm{n}]$ stands for the pulse output of neuron and it gets either the binary value 0 or 1 .

\section{c) The Intersecting Cortical Model (ICM)}

The ICM algorithm is especially designed for enhancing features without sharp edges or straight lines in images. The theoretical foundation of ICM is given and it is shown how the ICM can be derived as a reduced set of equations of the Pulse-Coupled Neural Network (PCNN) model based upon models proposed by Eckhorn and Reitboeck.

$$
\begin{aligned}
& F_{i, j}[n]=f F_{i, j}[n-1]+S_{i, j}+W_{i, j}\{Y[n-1]\} \\
& Y_{i, j}[n]= \begin{cases}1, & F_{i, j}[n]>\Theta_{i, j}[n-1] \\
0 & \text { otherwise }\end{cases} \\
& \Theta_{i, j}[n]=g \Theta_{i, j}[n-1]+h Y_{i, j}[n]
\end{aligned}
$$

In these equations, $\mathrm{S}[\mathrm{i}, \mathrm{j}]$ is the stimulus (the input image, scaled so that the largest pixel value is 1.0 ). $\mathrm{H}_{\mathrm{i}, \mathrm{j}}$ is defined by the threshold ofthe neuron and $\mathrm{Y}$ is the output image. $\mathrm{f}, \mathrm{g}$, and $\mathrm{h}$ are scalars. The ICM has higher speed because of reducing the number of equations over the PCNN. This model usually is applied in image segmentation, feature extraction, etc.

\section{d) Types of Filters}

Filters are used to remove noise from an image. The following are the filters that are used.

\section{Adaptive Median Filter}

The adaptive median filtering has been applied widely as an advanced method .The Adaptive median Filter performs spatial processing to determine which pixels in an image have been affected by impulse noise. It classifies pixels as noise by comparing each pixel in the image to its surrounding neighbor pixels.. Its main purpose is to remove impulse noise, smoothing other noise, reduce distortion, like excessive thinning or thickening of object boundaries. Its algorithm is given below:

Level A: A1 = Zmed $-\mathrm{Zmin}$

$\mathrm{A} 2=\mathrm{Zmed}-\mathrm{Zmax}$

if $\mathrm{A} 1>0$ AND $\mathrm{A} 2<0$, go to level $\mathrm{B}$ 
else increase the window size if window size $<$ Smax, repeat

level A

else output Zxy

Level B: B1 = Zxy - Zmin

B2 = Zxy - Zmax

if $\mathrm{B} 1>0$ AND B2 <0, output

Zxy

else output Zmed

where Zmin is minimum gray level value in Sxy,Zmax is maximum gray level value in Sxy, Zmed is median of gray levels in Sxy,Zxy is gray level at coordinates (x,y) and Smax is maximum allowed size of Sxy. The advantage of using adaptive filter is it preserves details of an image and smooths non-impulsive noise.

\section{Weiner Filter}

The Wiener filtering executes an optimal trade-off between inverse filtering and noise smoothing. It removes the additive noise and inverts the blurring simultaneously. The Wiener filtering is optimal in terms of the mean square error.. The orthogonality princip implies that the Wiener filter in Fourier domain can be expressed as follows:

$$
w\left(f_{1}, f_{2}\right)=\frac{H^{*}\left(f_{1}, f_{2}\right) S_{x x}\left(f_{1} f_{2}\right)}{\left|H\left(f_{1} f_{2}\right)\right|^{2} S_{x x}\left(f_{1} f_{2}\right)+S_{h \eta}\left(f_{1} f_{2}\right)^{\prime}}
$$

Where $S_{x x}(f 1, f 2), S_{\eta \eta}(f 1, f 2)$ are respectively power spectra of the original image and the additive noise, and is the blurring filter The major advantage of using this filter is that it controls output error and straightforward to design

\section{Bilateral Filtering}

The bilateral filter is a non-linear technique that can blur an image while respecting strong edges. Its ability to decompose an image into different scales without causing haloes after modification has made it ubiquitous in computational photography applications such as tone mapping, style transfer, relighting, and denoising. Its formulation is simple,each pixel is replaced by a weighted average of its neighbors. The bilateral filter is defined as

$$
I^{\text {filtered }}(x)=\frac{1}{W_{p}} \sum_{x_{i} \in \Omega} I\left(x_{i}\right) f_{r}\left(\left\|I\left(x_{i}\right)-I(x)\right\|\right) g_{s}\left(\left\|x_{i}-x\right\|\right)
$$

where $\mathrm{I}^{\mathrm{filtered}}$ is the filtered image, $\mathrm{I}$ is the original input image to be filtered, $\mathrm{x}$ are the coordinates of the current pixel to be filtered, $\Omega$ is the window centered in $x, f_{r}$ is the range kernel for smoothing differences in intensities., $g_{s}$ is the spatial kernel for smoothing differences in coordinates. The main advantage of bilateral filter is that its averaging is good to remove random noise

\section{VI.EXPERIMENTAL RESULT}

TABLE 1: VARYING LOG VALUES

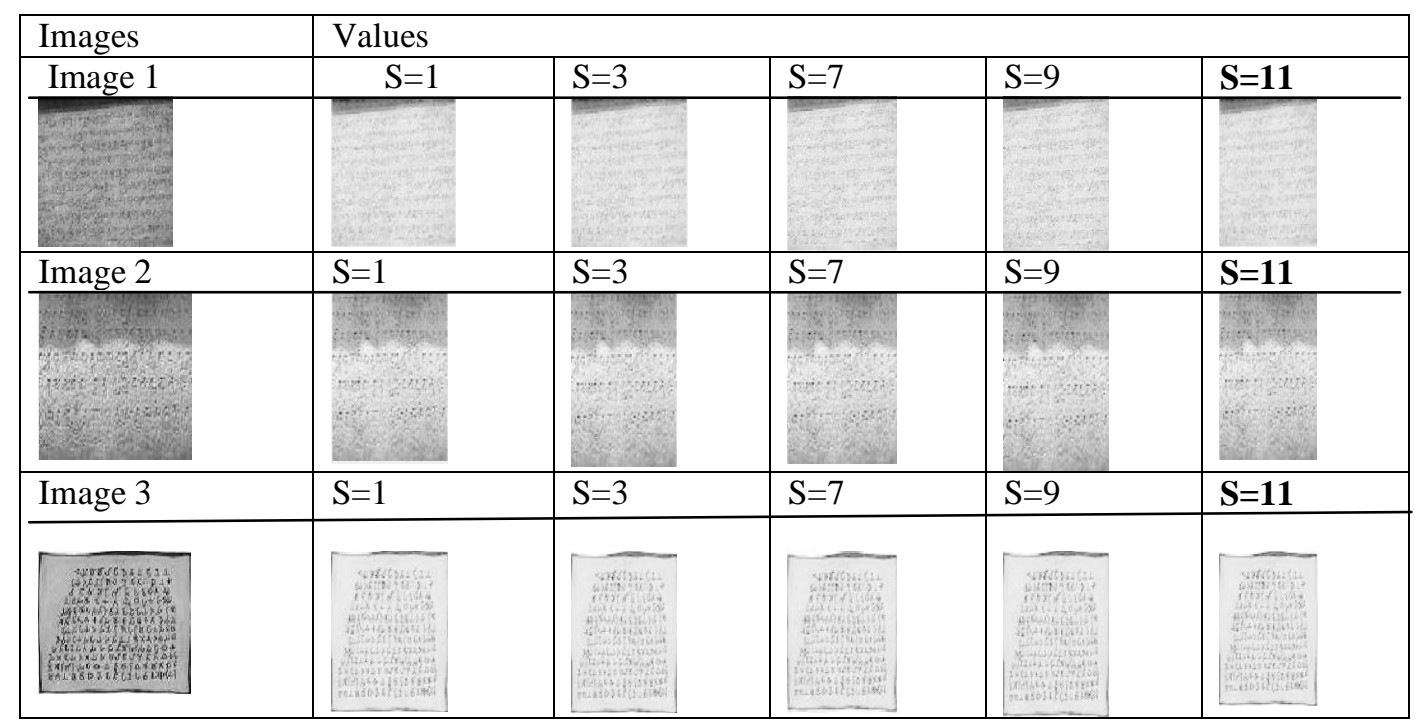


Experiments were conducted to improve the quality of a contaminated brahmi script taken as an input. Log values is applied to the script to highlight the minor details of an image is shown above in Table 1.

To the high contrast value of log applied to an image, Pulse Coupled Neural Network (PCNN) Intersecting Cortical Model (ICM) and different filtering techniques such as Adaptive Median Filter, Weiner Filter and Bilateral Filter are applied is shown below in Table 2

\section{TABLE 2: TO THE LOG VALUE S=11 ICM AND FILTERS ARE APPLIED}

\begin{tabular}{|c|c|c|c|}
\hline Images & Adaptive & Wiener & Bilateral \\
\hline Image1 & 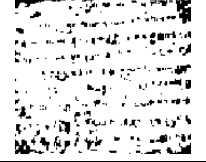 & 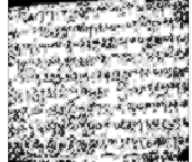 & 8 \\
\hline Image 2 & atsos & $\begin{array}{l}0 \\
4 \\
4\end{array}$ & Bastos \\
\hline Image 3 & 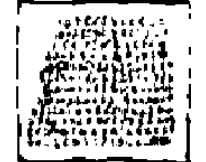 & 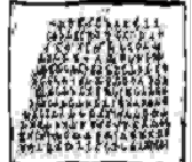 & | \\
\hline
\end{tabular}

VII.PERFORMANCE EVALUATION

\section{A. Performance Metrics}

The Performance metrics is used to evaluate the performance of the improved quality image. The performance metrics used in the paper are PSNR, SSIM, RMSE, EPI, COC [14]. In this paper an additional metric UQI is used with the other metrics.

\section{Peak-Signal to Noise-Ratio(PSNR)}

PSNR, is the ratio between the maximum possible power of a signal and the power of corrupting noise. It is computed as:

$$
\text { PSNR=20 } \log 10(255 / \text { RMSE })
$$

Higher PSNR generally indicates the image is of higher quality.

\section{Structural Similarity Index Measure (SSIM)}

The Structural Similarity (SSIM) index measure is a method for measuring the similarity between two images. It is computed as:

Where $\mu_{\mathrm{x}}=\sum_{\mathrm{i}=1}^{\mathrm{N}} \mathrm{W}_{\mathrm{i}} \mathrm{X}_{\mathrm{i}}$

$$
\operatorname{SSIM}(x, y)=\frac{\left(2 \mu_{X} \mu_{Y}+C 1\right)\left(2 \sigma_{X Y}+C 2\right)}{\left(\mu^{2}{ }_{X}+\mu^{2}{ }_{Y}+C 1\right)\left(\sigma^{2}{ }_{X}+\sigma^{2}{ }_{Y}+C 2\right)}
$$

$\left.\sigma_{\mathrm{x}}=\sum_{\mathrm{i}=1}^{\mathrm{N}} \mathrm{W}_{\mathrm{i}}\left(\mathrm{x}_{\mathrm{i}}-\mu_{\mathrm{x}}\right)^{2}\right)^{\frac{1}{2}}$

$\sigma_{\mathrm{xy}}=\sum_{\mathrm{i}=1}^{\mathrm{N}}\left(\mathrm{x}_{\mathrm{i}}-\mu_{\mathrm{x}}\right)\left(\mathrm{y}_{\mathrm{i}}-\mu_{\mathrm{y}}\right)$

$\mathrm{C}_{1}=\left(\mathrm{K}_{1}, \mathrm{~L}\right)^{2}$ and $\mathrm{C}_{2}=\left(\mathrm{K}_{2}, \mathrm{~L}\right)^{2}$

Where $\mathrm{L}$ is the range of pixel values( 255 for 8 -bit grayscale images). And $\mathrm{K}_{1} \ll<1$ is a small constant and also $\mathrm{K}_{2} \ll<1$.

\section{Root Mean Square Error (RMSE)}

The square root of the mean/average of the square of all of the error. The use of RMSE is very common and it makes an excellent general purpose error metric for numerical predictions. Compared to the similar Mean Absolute Error, RMSE amplifies and severely punishes large errors.

$$
\operatorname{RMSE}=\sqrt{\frac{1}{\mathrm{n}} \sum_{\mathrm{i}=1}^{\mathrm{n}}\left(\mathrm{y}_{\mathrm{i}}-\hat{\mathrm{y}}_{\mathrm{i}}\right)^{2}}
$$

Where $y_{i}$ is original image and $\hat{y}_{i}$ is an improved quality image. 


\section{Edge Preservation Index (EPI)}

EPI are widely used as useful tools for a variety of image editing and manipulation tasks, while preserving fine details and geometrical structures in the original image. It is computed according to equation

$$
\mathrm{EPI}=\frac{\sum(\Delta \mathrm{x}-\overline{\Delta \mathrm{x}})(\Delta \mathrm{y}-\overline{\Delta \mathrm{y}})}{\sum(\Delta \mathrm{x}-\overline{\Delta \mathrm{x}})^{2}(\Delta \mathrm{y}-\overline{\Delta \mathrm{y}})^{2}}
$$

where, $\Delta \mathrm{x}$ and $\Delta \mathrm{y}$ are the high pass filtered versions of images $\mathrm{x}$ and $\mathrm{y}$, obtained with a $3 \times 3$ pixel standard approximation of the Laplacian operator. The $\Delta \mathrm{x}$ and $\Delta \mathrm{y}$ are the mean values of the high pass filtered versions of $\Delta \mathrm{x}$ and $\Delta \mathrm{y}$ respectively.

\section{Correlation Coefficient (CoC)}

The correlation coefficient is a measure that determines the degree to which two variables' movements are associated. The range of values for the correlation coefficient is -1.0 to 1.0. If a calculated correlation is greater than 1.0 or less than -1.0 , a mistake has been made. A correlation of -1.0 indicates a perfect negative correlation, while a correlation of 1.0 indicates a perfect positive correlation.

$$
\rho_{\mathrm{xy}}=\operatorname{cov}\left(\mathrm{r}_{\mathrm{x}, \mathrm{r}} \mathrm{r}_{\mathrm{y}}\right.
$$

\section{Universal Quality Index(UQI)}

The universal objective image quality index, is easy to calculate and applicable to various image processing applications. Instead of using traditional error summation methods, this index is designed by modeling any image distortion as a combination of three factors: loss of correlation, luminance distortion, and contrast distortion. This is given as follows:

$$
Q=\frac{4 \sigma_{x y} \bar{x} \bar{y}}{\left(\sigma_{x}^{2}+\sigma_{y}^{2}\right)\left[(\bar{x})^{2}+(\bar{y})^{2}\right]},
$$

where

$$
\begin{gathered}
\bar{x}=\frac{1}{N} \sum_{i=1}^{N} x_{i}, \quad \bar{y}=\frac{1}{N} \sum_{i=1}^{N} y_{i}, \\
\sigma_{x}^{2}=\frac{1}{N-1} \sum_{i=1}^{N}\left(x_{i}-\bar{x}\right)^{2}, \quad \sigma_{y}^{2}=\frac{1}{N-1} \sum_{i=1}^{N}\left(y_{i}-\bar{y}\right)^{2} \\
\sigma_{x y}=\frac{1}{N-1} \sum_{i=1}^{N}\left(x_{i}-\bar{x}\right)\left(y_{i}-\bar{y}\right) .
\end{gathered}
$$

The dynamic range of $\mathrm{Q}$ is $[-1,1]$. The best value of 1 is achieved if and only if $\mathrm{Y}_{\mathrm{I}}=\mathrm{x}_{\mathrm{I}}$.

\section{B. Performance Evaluation}

The performance of the script with ICM and filtering techniques were examined using the metrics PSNR, SSIM, RMSE, EPI CoC and UQI. The experiments are performed on image1, image 2 and image 3 with ICM and the three filtering techniques and the results are shown below in the Table 3.

Table 3: Metrics For The Enhanced Image After Applying Filters

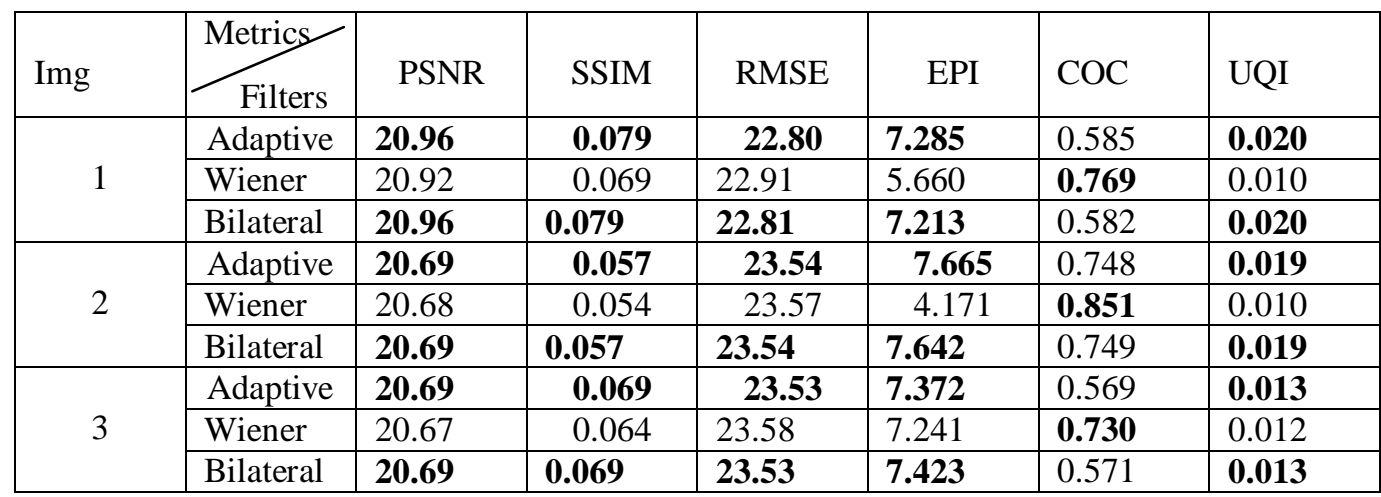




\section{CONCLUSION}

The images are compared by varying log transformation values to find an enhanced image. Filters like Adaptive, Wiener and Bilateral are used for all the enhanced images from which metrics like Peak-Signal to Noise Ratio (PSNR), Structural Similarity Index Measure (SSIM), Root Mean Square Error (RMSE), Edge Preservation Index (EPI),Correlation Coefficient (CoC) and Universal Quality Index(UQI) are calculated. It is found that in all the images 1,2 and 3 Adaptive and Bilateral filter shows better results in enhancing images with increased values for the metrics PSNR ,SSIM, EPI, and UQI works better than all the other metrics. RMSE. Shows decreased value. Bilateral filter shows a better result in preserving the edges of the images. Wiener Filter shows a better result for the Correlation Coefficient.

\section{REFERENCES}

[1] Sasi Gopalan, Madhu S Nair and Souriar Sebastian “Approximation Studies on Image Enhancement Using Fuzzy Technique" International Journal of Advanced Science and Technology, Vol. 10, pp.11-26, September, 2009.

[2] Bhabatosh Chanda and Dwijest Dutta Majumder, 2002, Digital Image Processing and Analysis.

[3] R. Jain, R. Kasturi and B.G. Schunck, Machine Vision, McGraw-Hill International Edition, 1995.

[4] Papiya C. Histogram equalization by cumulative frequency distribution. International Journal of Scientific and Research Publications. 2012; 2(7):1-4.

[5] R. Eckhorn, H.J. Reitboeck, M. Arndt, P.W. Dicke, A neural network for feature linking via synchronous activity: results from cat visual cortex and from simulations, in: Models of Brain Function, Cambridge University Press, Cambridge, UK, 1989, pp. 255-272.

[6] H.J. Reitboeck, R. Eckhorn, M. Arndt, P. Dicke, A model of feature linking via correlated neural activity, in: Synergistics of Cognition, Springer, New York, 1989, pp. 112-125

[7] R. Eckhorn, H.J. Reitboeck, M. Arndt, P.W. Dicke, Feature linking via synchronization among distributed assemblies: simulation of results from cat cortex, Neural Computing 2 (1990) 293-307.

[8] I.A. Rybak, N.A. Shevtsova, L.N. Podladchikova, A.V. Golovan, A visual cortex domain model and its us for visual information processing, Neural Networks4 (1991) 3-13.

[9] I.A. Rybak, N.A. Shevtsova, V.M. Sandier, The model of a neural network visual preprocessor, Neurocomputing 4 (1992) $93-102$.

[10] J.L. Johnson, D. Ritter, Observation of periodic waves in a pulse-coupled neural network, Optical Letter 18 (1993) 1253.

[11] H.S. Ranganath, G. Kuntimad, J.L. Johnson, Pulse coupled neural networks for image processing, in: Proc. of the Southeast Conference on 'Visualize the future', 1995, pp. 37-43.

[12] L. Johnson, H. Ranganath, G. Kuntimad, H.J. Caulfield, Pulse-coupled neural networks, in: Neural Network and Pattern Recognition, Academic Press, SanDiego, CA, 1998, pp. 1-56.

[13] Hodgkin, A. L., \& Huxley, A. F. (1952). A quantitative description of membrane current and its application to conduction and excitation in nerve. Journal of Physiology, 117,500-544.

[14] S.Ashika Parvin \& S.Shajun Nisha. "Historical Document Preservation Using ICM And Filters". International Journal of Innovative Research in Computer and Communication Engineering, vol.4, Issue 12, December 2016.

\section{BIOGRAPHIES}

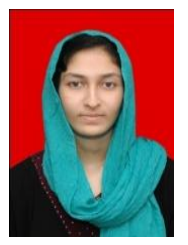

S. Ashika Parvin is currently pursuing M.Phil degree in computer science in Sadakathullah Appa College, Tirunelveli. She has done her MCA degree in Computer Science from ST. Xavier's College, Tirunelveli and BCA degree in Computer Science from Sadakathullah Appa College, Tirunelveli under Manonmaniam Sundaranar University, Tirunelveli.

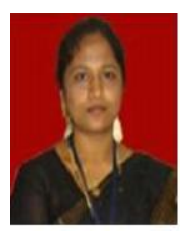

Shajun Nisha S. Professor and Head of the Departmen of PG Computer Science, sadakathullah Appa College, Tirunelveli. She has completed M. Phil(Computer Science) and M.Tech(Computer and Information Technology) in Manonmaniam Sundaranar University, Tirunelveli. She has involved in various academic activities. She has attended so many national and International seminars, Conferences and presented numerous research papers. She is a member of ISTE and IEANG and her specialization is Image Mining

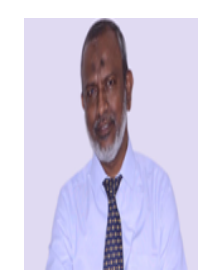

Dr. M. Mohamed Sathik M.Tech., M.Phil., M.Sc., M.B.A., M.S., Ph.D has so far guided more than 35 research scholars. He has published more than 100 papers in International Journals and also two books. He is a member of curriculum development committee of various universities and autonomous colleges of Tamil Nadu.His specializations are VRML, Image Processing and Sensor Networks. 